\title{
Telescoping bronchial anastomosis for extended sleeve lobectomy
}

\author{
Shinichiro Miyoshi, MD, PhD, Motohiko Tamura, MD, PhD, Osamu Araki, MD, \\ Naoko Yoshii, MD, PhD, Yoko Karube, MD, PhD, Norio Seki, MD, Hideo Umezu, MD, \\ Satoru Kobayashi, MD, PhD, Hiromi Ishihama, MD, Sensuke Nagai, MD, PhD, \\ and Noriyoshi Sawabata, MD, PhD, Tochigi, Japan
}

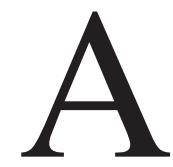

$\mathrm{n}$ extended sleeve lobectomy is rarely attempted to avoid a pneumonectomy for patients with primary lung cancer. ${ }^{1}$ This atypical bronchoplasty procedure is technically demanding because there is a large size discrepancy between the 2 bronchial stumps. Herein we report successful implementation of an extended sleeve lobectomy with a telescoping anastomosis.

\section{Clinical Summary}

A 76-year-old man was referred to our hospital for additional investigation of an abnormal shadow on chest roentgenographic images obtained during a screening examination. A computed tomographic scan demonstrated a large mass shadow, $4 \times 5 \mathrm{~cm}$ in size, that originated in the posterior segment of the right upper lobe (S2; Figure 1, $A$ and $B$ ). Flexible fiberoptic bronchoscopy revealed a tumor that had completely occluded the posterior segmental bronchus of the right upper lobe (B2). A biopsy specimen was diagnosed as squamous cell carcinoma of the lung. Magnetic resonance imaging of the brain, computed tomographic scanning of the abdomen, and a bone scintigram demonstrated no evidence of distant metastases. Thus his clinical stage was classified as T2 N0 M0, stage IB disease.

The chest was opened through the fifth intercostal space with a posterolateral thoracotomy after achievement of general anesthesia by using a double-lumen endotracheal tube. The tumor originating in S2 had invaded the outer wall of the intermediate bronchus (Figure 1, C). A recurrent branch of the pulmonary artery to the posterior segment of the upper lobe (A2b) and a branch of the pulmonary artery to the superior segment of the lower lobe (A6) were not isolated because of tumor invasion (Figure $1, D$ ). The pulmonary artery branches of the upper lobe and middle lobe, except for $A 2 b$, as well as the superior pulmonary vein, were double

From the Department of Cardiothoracic Surgery, Dokkyo University School of Medicine, Tochigi, Japan.

Received for publication April 27, 2006; revisions received May 1, 2006; accepted for publication May 17, 2006.

Address for reprints: Shinichiro Miyoshi, MD, PhD, Department of Cardiothoracic Surgery, Dokkyo University School of Medicine, 880 Kitakobayashi, Mibu, Tochigi, 321-0293, Japan (E-mail: miyoshi@ dokkyomed. ac.jp).

J Thorac Cardiovasc Surg 2006;132:978-80

$0022-5223 / \$ 32.00$

Copyright $\odot 2006$ by The American Association for Thoracic Surgery doi:10.1016/j.jtcvs.2006.05.055 ligated and divided. After administration of heparin, the main and basal pulmonary arteries were clamped and divided at the inner side of each clamp. The tumor was extirpated with division of the

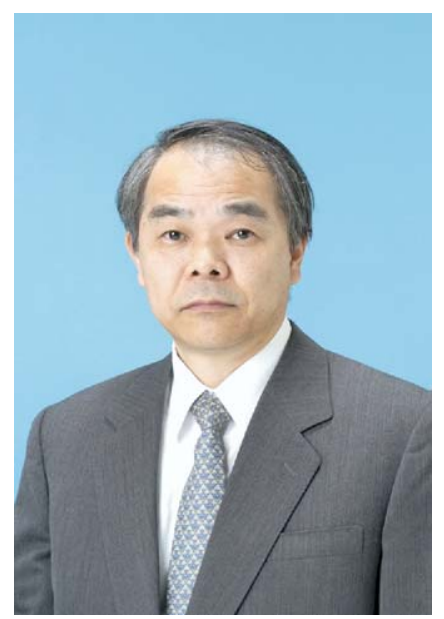

Dr Miyoshi main and basal bronchi and a segmentectomy of S6.

There was a large size discrepancy between the bronchial stumps, and thus 2 adjusting stitches were placed in the membranous part of the main bronchial stump and tied as shown in Figure 2, A and B. A telescoping bronchial anastomosis was then performed with interrupted sutures ${ }^{2}$ by using $4-0$ monofilament absorbable materials. The cartilage sutures were tied so that the basal bronchus was inserted into the main bronchus at a depth equal to the circumference of the cartilage. A pericardial incision was performed around the inferior pulmonary vein to decrease tension on the bronchial anastomosis. A pulmonary artery anastomosis was also performed, after placing an adjusting running suture onto the proximal stump. The anastomoses of the bronchus and pulmonary artery were completed as shown in Figure 2, $C$. Finally, the pericardial fat pad was inserted between the bronchial and pulmonary artery anastomoses.

The postoperative course was uneventful. Flexible fiberoptic bronchoscopy was performed 10 months after the operation, which showed no stenosis at the anastomotic site (Figure 2,D). The patient was doing well without recurrence 3 years and 7 months after the operation.

\section{Discussion}

Recently, Okada and colleagues ${ }^{1}$ reported the results of extended sleeve lobectomy procedures for 15 patients who were classified into 3 groups on the basis of the mode of reconstruction: group A, anastomosis between the right main and lower bronchi or basal segmental bronchi with resection of the upper and middle lobes or those and S6; group B, anastomosis between the left main and basal segmental bronchi with resection of the upper lobe and S6; and group $\mathrm{C}$, anastomosis between the left main and upper division bronchi with resection of the lingular segment and lower lobe.

There are 3 techniques used for size matching between the proximal and distal bronchial stumps. One is to use 2 adjusting stitches in the membranous part of the larger stump. ${ }^{1}$ The second technique is to make a cuff on the smaller stump by trimming the carina. We have applied the second technique to patients in group $\mathrm{C}$ according to the above classification. The third technique is a telescoping anastomosis. Our experience has shown that the largest difference in size between the 2 bronchial stumps occurs in patients classified as group A, as in 

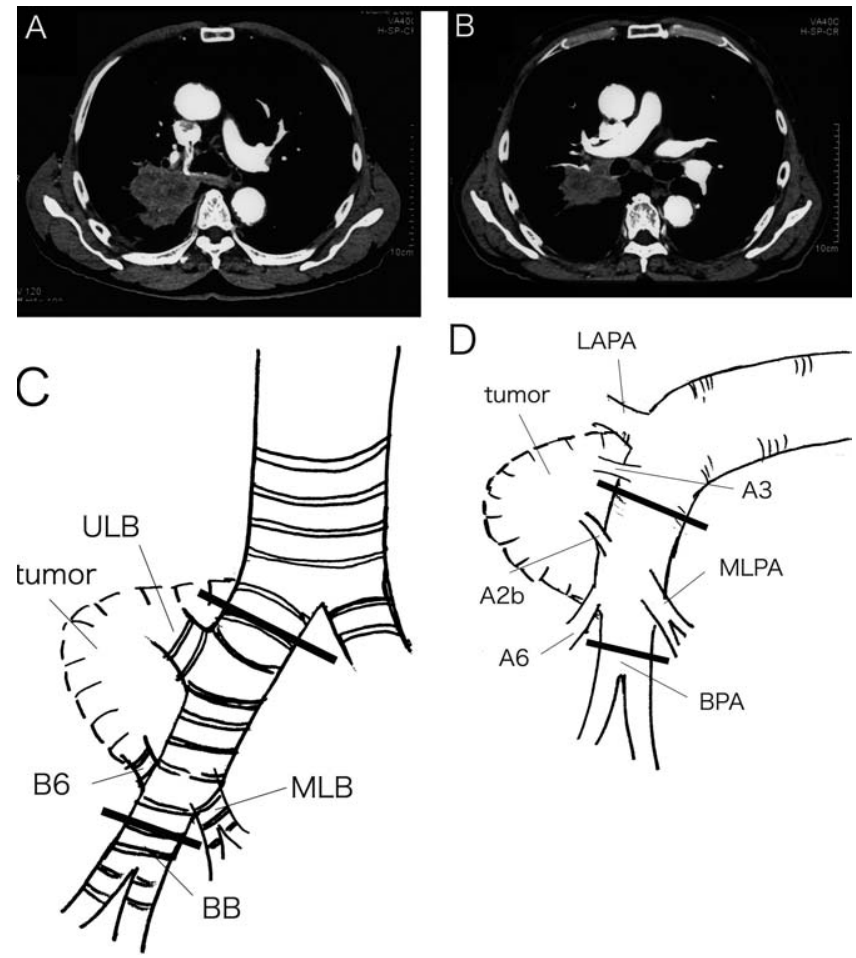

Figure 1. Appearance of tumor on computed tomographic scan and operative findings. A and B, Computed tomographic images show a large mass shadow, $4 \times 5 \mathrm{~cm}$ in size, in the posterior segment of the upper lobe (S2) extending down to the lower lobe. C, A tumor originating in S2 is shown invading the outer wall of the intermediate bronchus. D, A recurrent branch of the pulmonary artery to the posterior segment of the upper lobe (A2b) and a branch of the pulmonary artery to the superior segment of the lower lobe (A6) show tumor invasion. Heavy solid lines in $\mathrm{C}$ and D demonstrate divided sites. ULB, Upper lobe bronchus; MLB, middle lobe bronchus; $B 6$, superior segmental bronchus of the lower lobe; $B B$, basal bronchus of the lower lobe; $L A P A$, large anterior pulmonary arterial branch of the upper lobe; $A 2 b$, recurrent pulmonary arterial branch to the posterior segment of the upper lobe; A6, pulmonary arterial branch to the superior segment of the lower lobe; $A 3$, pulmonary arterial branch to the anterior segment of the upper lobe; MLPA, middle lobe pulmonary artery; $B P A$, basal segmental pulmonary arterial branch. the present case, probably because the diameter of the right main bronchus is generally larger than that of the left main bronchus. The distal stump is also quite thin and frail. Despite these disadvantages for a bronchial anastomosis with an extended sleeve lobectomy, a telescoping-type anastomosis might reduce anastomosis-related complications. This technique is also useful for single-lung transplanta-
A
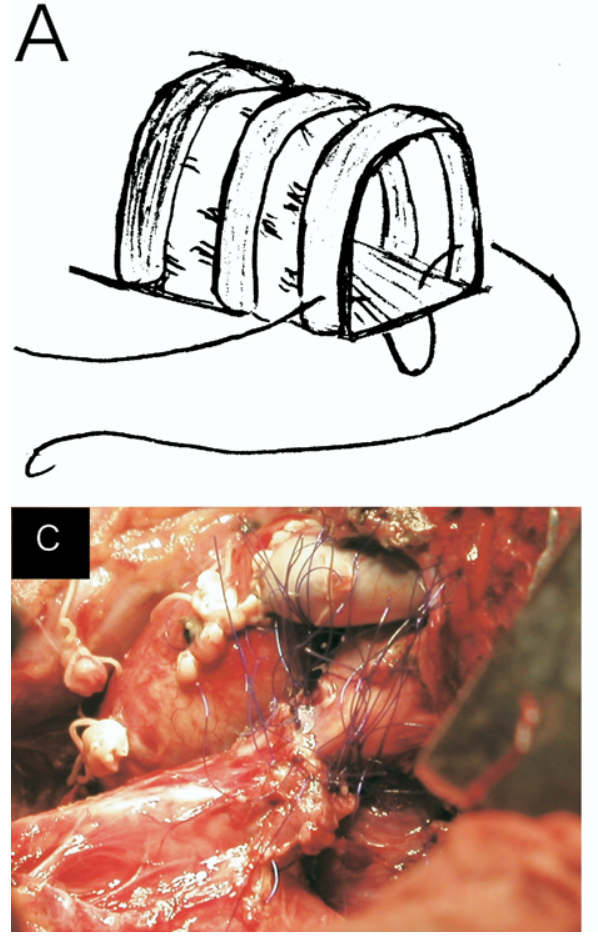
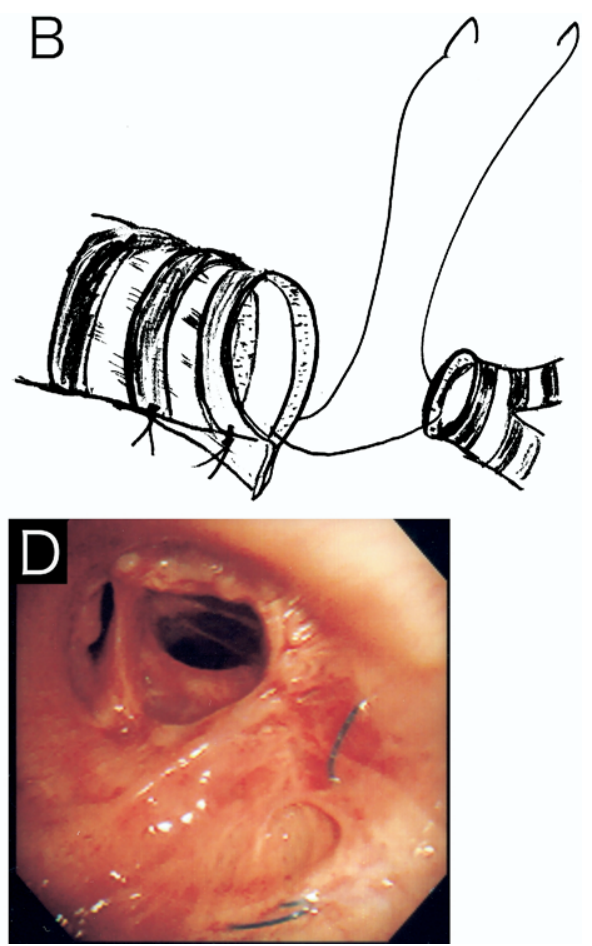

Figure 2. Appearance of bronchial anastomosis. A and B, Two stitches were used in the membranous part of the main bronchial stump, and then a telescoping bronchial anastomosis was performed with interrupted sutures. C, Completed anastomoses of the bronchus and pulmonary artery are shown. D, Flexible fiberoptic bronchoscopic image shows the anastomotic site 10 months after the operation. 
tion $^{3}$ or bronchoplasty procedures in patients with lung cancer after induction therapy ${ }^{2}$ for the same purpose.

\section{References}

1. Okada M, Tsubota N, Yoshimura M, Miyamoto Y, Matsuoka H, Satake S, et al. Extended sleeve lobectomy for lung cancer: the avoidance of pneumonectomy. J Thorac Cardiovasc Surg. 1999;118: 710-4.

2. Ohta M, Sawabata N, Maeda H, Matsuda H. Efficacy and safety of tracheobronchoplasty after induction therapy for locally advanced lung cancer. J Thorac Cardiovasc Surg. 2003;125:96-100.

3. Calhoon JH, Grover FL, Gibbons WJ, Bryan CL, Levine SM, Bailey SR, et al. Single lung transplantation: alternative indications and technique. J Thorac Cardiovasc Surg. 1991;101:816-25.

\title{
Simultaneous heart valve replacement and reconstruction of the radiation-damaged chest wall with a delayed vertical rectus abdominis myocutaneous flap
}

\author{
Thomas Strecker, MD, ${ }^{a}$ Richard Feyrer, MD, ${ }^{a}$ Raymund E. Horch, MD, ${ }^{b}$ Michael Weyand, MD, ${ }^{a}$ and Ulrich Kneser, MD, \\ Erlangen, Germany
}

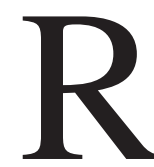
adiation ulcer of the chest wall after breast cancer treatment represents a severe physiologic and psychological problem. Patients with radiation ulcer who require cardiac surgery are at high risk for development of consecutive sternum osteomyelitis. In this article, we report a successful surgical aortic valve replacement and mitral valve reconstruction in combination with simultaneous reconstruction of the radiation-damaged chest wall using a delayed superiorly based vertical rectus abdominis myocutaneous flap in a 71-year-old patient. Cardiac surgery and plastic surgical reconstruction of the chest wall in a joint approach at the same time allow for safe and efficient treatment and may help to reduce the total number of operations and hospital stays.

\section{Clinical Summary}

A 71-year-old white woman with suspected endocarditis and symptoms of heart failure was referred to the cardiology department of our hospital. She noted progressive dyspnea, arterial hypertension, fatigue, and exhaustion. Twenty years ago, a right-sided radical mastectomy, followed by irradiation of the chest wall, was performed for treatment of breast cancer. Five

\footnotetext{
From the Center of Cardiac Surgery a and the Department of Plastic and Hand Surgery, ${ }^{\mathrm{b}}$ Friedrich-Alexander-University of Erlangen-Nuremberg, Erlangen, Germany,

Received for publication March 18, 2006; revisions received April 22, 2006; accepted for publication May 17, 2006.

Address for reprints: Thomas Strecker, MD, Center of Cardiac Surgery, Friedrich-Alexander-University of Erlangen-Nuremberg, Krankenhausstr 12, D-91054 Erlangen, Germany (E-mail: thomas.strecker@ herz.imed.unierlangen.de)

J Thorac Cardiovasc Surg 2006;132:980-2

$0022-5223 / \$ 32.00$

Copyright $\odot 2006$ by The American Association for Thoracic Surgery doi:10.1016/j.jtcvs.2006.05.063
}

years later, progressive skin fibrosis developed. Within the last 7 years before admission, the patient reported recurrent parasternal skin ulcerations with fistula.

At admission to the hospital, the laboratory tests showed a leukocytosis of $11.42 \times 10^{3} / \mu \mathrm{L}$ (normal range, $<10 \times 10^{3} / \mu \mathrm{L}$ ), and the other values were within the normal range. Physical examination revealed a $13 \times 9-\mathrm{cm}$ parasternal unstable scar with skin atrophy (Figure 1). The skin was attached to the rib cage because the pectoralis major muscle had been resected previously. On admission, there was no fistula visible. Transesophageal echocardiography revealed a severe aortic valve stenosis with maximum/mean pressure gradients of 74/43 $\mathrm{mm} \mathrm{Hg}$ and an aortic valve orifice of $0.84 \mathrm{~cm}^{2}$. Furthermore, there was a mediocre mitral valve insufficiency. There were no signs of endocarditis. The cardiac catheterization showed no significant stenoses of the coronary arteries and an ejection fraction of $44 \%$. Radiography and magnetic resonance imaging of the sternum disclosed osteolysis of the central third of the sternum either caused by osteomyelitis or aseptic osteoradionecrosis and fibrosis of the surrounding soft tissue without any evidence of mediastinitis.

Seven days before cardiac surgery, a right-sided vertical rectus abdominis myocutaneous (VRAM) flap (dimension, $10 \times 26 \mathrm{~cm}$ ) was delayed. The skin island was incised, and the inferior epigastric artery was ligated (Figure 1). Seven days later, the patient was again taken to the operating theater, where a median sternotomy was performed, and cardiopulmonary bypass was installed through aorto-right atrium cannulation. The calcified aortic valve was excised and replaced with a biologic prosthesis with a $23-\mathrm{mm}$ diameter. This mitral valve insufficiency was away with implantation of an annuloplastic ring with a 28-mm diameter. Intraoperatively, there were no signs of any myocarditis, endocarditis, or sternum osteomyelitis, and therefore the chest was closed with cerclage. The unstable parasternal and presternal scar was excised, and the delayed superiorly based VRAM flap was completely raised and transferred into the defect. The inferior part of the anterior rectus sheath was reconstructed with a polypropylene surgical mesh, and wounds were closed in layers at the recipient and donor sites. 